\title{
Rapid Detection of Characters on Automobile Electronic Gear Lever
}

\author{
Yongli Zhang ${ }^{1,2, ~ *, ~ Y u a n s h e n g ~ Q i ~}{ }^{1,2}$, Ximu Make ${ }^{1,2}$ and Shilu Liu ${ }^{1,2}$ \\ ${ }^{1}$ School of Mechanical and Electrical Engineering, Beijing Institute Graphic Communication, Beijing, 102600, China \\ ${ }^{2}$ Intelligent Manufacturing Laboratory, Beijing Institute of Graphic Communication, Beijing, 102600, China
}

Received 2 July 2021; Accepted 14 September 2021

\begin{abstract}
Character display of automotive electronic gear lever is affected by LED lamp beads and transparent cover trim panel. The problems in the manufacturing processes include abnormal color coordinates, abnormal luminance value, and incomplete characters. The algorithm for rapid detection based on CMOS industrial cameras was proposed to replace manual visual inspection and improve abnormal recognition rate and detection efficiency. The color coordinates and luminance model for transient detection of the electronic gear lever were established through the temperature drift. The improved method of circle projection feature was used to achieve recognition. Curvature detection was used for incomplete detection of characters. The effectiveness was verified by experiments. Results show that high and low luminance weight traceability for color coordinate calibration has good consistency, and the luminance and gray values have an exponential relationship after the temperature drift of the characters. The method of circular projection feature has high robustness for the recognition of inclined and reduced character outlines. Curvature detection is effective for incomplete contours. The curvature steps of characters at different positions are six, four, and three, and the threshold is uniformly set to 0.15 . The outflow rate of the problem electronic gear lever is reduced, and the productivity is increased by 0.3 percent with this detection method. This study provides a reference for the test platform of other types of electronic gear lever and rapid detection of characters.
\end{abstract}

Keywords: Electronic gear lever, Character recognition, Defect detection, Color coordinates

\section{Introduction}

With the development of automobile industry, electronic gear lever has become increasingly popular in new energy vehicles. The largest difference between the electronic shifting system and the traditional shifting system is that the former uses an electronic shifting operating mechanism instead of the traditional shifting operating mechanism. At the same time, the operating panel accepts instructions from the central controller to light up the corresponding gear characters in different states. Different enterprises have different definitions of operation forms, including lever type, rotary/dial type, push switch type, and column lever type, all of which have the common point of visual display of gear operation results [1].

Generally, the characters on electronic gear lever are parking gear $(\mathrm{P})$, reverse gear $(\mathrm{R})$, neutral gear $(\mathrm{N})$, drive gear (D), and sports gear (S), and the displayed colors include white, green, and orange. The production and inspection process of the electronic gear lever integrates performance, appearance, color, and luminance information. At present, manual operation is often used to manually light each gear for visual inspection. Over fatigue causes low efficiency and high missed detection error rate. The luminance of the characters was disturbed not only by LED lamp beads, driving voltage, and current and transparent resin board, but also by the assembly environment in the workshop. Traditional visual inspection was performed for 30 minutes at a constant temperature in a laboratory, which was unsuitable for production transient inspection

*E-mail address: Ializhang@163.com

ISSN: 1791-2377 @ 2021 School of Science, IHU. All rights reserved.

doi:10.25103/jestr.144.11 phenomena. The flaw detection of CMOS industrial cameras becomes more mature and is gradually applied to different inspection occasions [2]. Developing a rapid inspection method and test system based on CMOS industrial cameras suitable for production is urgently required. For this reason, scholars have conducted a number of studies on the visual inspection, LED lamp bead color temperature, optical character recognition (OCR), character defect detection, and color coordinate calibration involved in the electronic gear lever $[3,7]$. However, the operation of electronic gear lever is fast, and the number of defective pictures is insufficient. The algorithm for the traditional template matching detection had poor detection accuracy and robustness. At the same time, it affected the detection efficiency and could not satisfy the requirements of production beat. As a result, improving the detection accuracy and efficiency is an urgent problem.

In this connection, a color coordinate and luminance model was established for transient detection of the electronic gear lever by using CMOS industrial cameras through the temperature drift traceability, and the algorithm for character defect detection was analyzed. The purpose was to improve the accuracy and inspection efficiency and provide a reference for rapid inspection system suitable for the production line of electronic gear lever.

\section{State of the art}

Scholars worldwide have conducted considerable exploration on the application of CMOS cameras [3,9]. Bingkai L [3] studied the dark current random signal of 
CMOS camera and analyzed the advantages of CMOS image sensor, such as high stability, low power consumption, and strong anti-interference from the mechanism, and even comparable to CCD sensors in some fields. Ayumi [4] also discussed the dark current, which was called the no luminance current, which referred to the unexpected charge generated by the camera image sensor under the condition of no luminance; the main reason affects the camera interference. These scholars provided a reference for CMOS cameras for luminance detection in darkrooms. Deng Wei [5] studied the photosensitive formula of CMOS image sensor, which provided a reference for the realization of luminance detection with CMOS camera and pointed out that cooling can reduce the random interference noise of the camera. The automatic exposure problem of digital cameras was discussed by Bernacki J [6]. He pointed out the problem of excessive exposure. The algorithm for automatic exposure, from which the camera depends, was expressed according to the image acquisition frame rate; however, it could not minimize the exposure area. Guo, Z. H. [7] proposed the algorithm for automatic exposure based on luminance histogram and region segmentation. The algorithm used the relationship between the time $\mathrm{f}$ of collecting a frame of image and the exposure time $t$ of CMOS camera; it realized exposure compensation by using automatic exposure to automatic gain. The algorithm could be used in various environments, but it consumed considerable time. Sohaib A [8] and Bai Y [9] pointed out that the three parameters affecting automatic exposure were aperture size, exposure time, and gain. The camera automatic exposure was determined by exposure time and gain when the aperture value was fixed. This condition provided a reference for establishing luminance model with fixed exposure time and gain in darkroom. Yan Limin [10] made an imaging luminance meter with a camera to measure the luminance on the LED display screen. The results show that the requirements of actual engineering were satisfied, but the measurement results were far from accurate with the measured values obtained by traditional methods. Thus, further improvement is necessary. Huang C. [11] considered the difference in color bands in the light environment to calibrate the camera chromaticity and then the chromaticity tristimulus value. The accuracy of luminance measurement was improved, and the luminance measurement of marine light environment was analyzed. Although this method was suitable for calibration in a large background environment, it was unnecessarily suitable for measurement in the darkroom. Tang Jialin [12] used the gray world method to solve the white balance noise of the CMOS cameras, approached the target in an exhaustive manner, and obtained a better color balance effect. Although the calibration effect was good, the amount of calculation was complicated. With deep convolutional neural network, Huang C. Q. [13] introduced intelligent classifier into traditional white balance method and obtained good convergence by using 34,200 pictures as training data. However, the training data of the picture library were extremely large to be suitable for small sample detection.

The algorithm for contour recognition is applied to the intelligent algorithm processing recently $[14,15]$. Sergio $M$ [14] proposed an end-to-end automatic license plate recognition (ALPR) method based on hierarchical convolutional neural network $(\mathrm{CNN})$ to realize the OCR character recognition of license plates in video streams. This algorithm considered synthetic and enhanced data to process limited training data. This algorithm had greater improvement and more suitable for processing pictures in the video stream than the traditional algorithm. With Alex Net deep learning network, Lee S [15] recognized the vehicle license plate in an offline state and applied it to the vehicle system. These intelligent algorithms required a large number of image training data, and the recognition accuracy was insignificantly improved with limited data, simple background, regular character shape, and single category. Li Yan [16] proposed a license plate recognition method based on scale-invariant feature transform (SIFT) matching. Contour recognition was conducted with the superresolution interpolation image, which improved recognition rate and calculation. Wen S.S. [17] explored on AGV character recognition based on improved $\mathrm{Hu}$ moment calculation combined with machine learning to improve accuracy. However, the approach did not obtain good robustness for the recognition and extraction of character outlines with rotation angles. Lin Y.H. [18] proposed a template matching method of circle projection feature (CPF) that was invariant to image translation, rotation, and scaling. However, it was suitable for flat picture characters and had good reference for surface character recognition.

These studies mainly focused on the calibration of CMOS camera and the algorithm for image recognition based on image defect library. Detection of the luminance of the characters on the electronic gear lever is rare, especially the condition of temperature drift. The gray value was extracted based on the RGB color gamut of CMOS industrial cameras, and the color coordinate and luminance model for transient detection of the electronic gear lever was established by the method of temperature drift tracing. The character contour was extracted by the improved algorithm of circular projection feature (IACPF) and analyzed by the algorithm for incomplete curvature detection to improve the collimation rate and the detection efficiency. It provides a reference for a rapid inspection system suitable for the production line of electronic gear lever.

The remainder of this study is organized as follows. Section 3 establishes the color coordinates and luminance model and proposes the method of circle projection feature. Section 4 discusses the applicability of the method through case studies. Section 5 summarizes the conclusions.

\section{Methodology}

In accordance with the product's physical model, the test framework was built and the test conditions and environment were provided. In addition, the color coordinate correction model was established. Initially, the dynamic white balance was carried out to correct the high and low luminance traceability calibration algorithm for the standard laboratory luminance instrument. Then, the instantaneous luminance model was established based on color coordinate correction, the gray value was calculated, and the test data of the luminance meter after 30 minutes were calibrated. Finally, the instantaneous luminance model was established by nonlinear mapping between the gray value and luminance value. The algorithm for circle projection feature was improved to be suitable for surface character detection and reduce the amount of computation. The maximum and minimum values were calculated, and then the radius was divided equally according to the equidistant ring. On the basis of the improved circular projection feature algorithm, the character contour base was recognized for contour defect 
detection, and the algorithm for curvature detection was used to detect the outer contour.

\subsection{Physical structure model of electronic gear lever}

The object of this study is the electronic gear lever of a brand of FAW Volkswagen produced by an auto part supplier. The electronic gear lever (Fig. 1) includes parking gear $(\mathrm{P})$, reverse gear $(\mathrm{R})$, neutral gear $(\mathrm{n})$, forward gear $(\mathrm{D} /$ $\mathrm{s})$, motion adjustment gear $(+-)$, and display symbol $(\nabla)$.

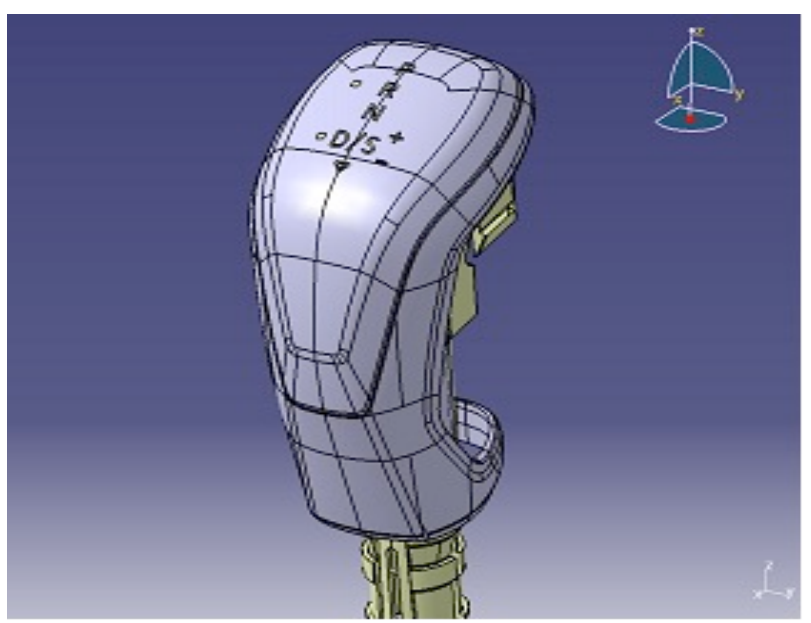

Fig. 1. Physical structure model of electronic gear lever

The six types of gear character states are low light, $\mathrm{P}$ high light, $\mathrm{R}$ high light, $\mathrm{N}$ high light, D/S high light, and +high light. The main factors that affect the luminance of characters are LED lamp beads, drive voltage, drive current, and light-transmitting resin plate. The impact of LED lamp beads and light-transmitting resin plate is the most serious. The outer contours of the characters are realized by laser engraving on the back of the resin plate coated with black lacquer. Defects, such as uneven thickness of the transparent part, incomplete characters, and black-and-white impurity points, were observed due to abnormal engraving or inappropriate cleaning.

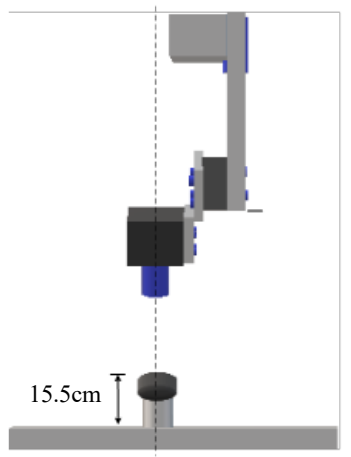

(a)

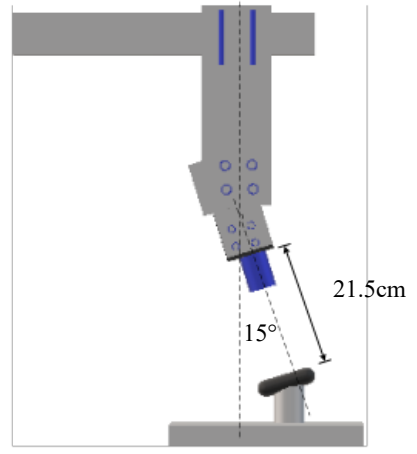

(b)
Fig. 2. Installation position diagram of electronic gear lever and the industrial camera. (a) Front view. (b) Side view

The height of electronic gear lever was $15 \mathrm{~cm}$, and the surface was an arc surface with an inclination of $15^{\circ}$ to the horizontal plane. The industrial camera was tilted $15^{\circ}$ and installed facing the arc surface of electronic gear lever to facilitate the robot to grasp, with a distance of $21 \mathrm{~cm}$ (Fig. 2).

The test environment was in a dark room. The test equipment included lumicam 1300 luminance colorimeter, wp-uc200 camera, m2518-mpw2 lens, self-made online current detector, and an automobile electronic gear lever. The computer operating system was Windows 10, the memory size was $8 \mathrm{G}$, and the processor model was Intel(R) Core (TM) i5-7200U. Image processing development tools included Visual Studio 2015, OpenCV image processing toolkit, and MATLAB R 2018a.

\subsection{Color coordinate correction model}

White balance restores the actual color of the image obtained under various light conditions by adjusting the proportional relationship of $\mathrm{R}, \mathrm{G}$, and $\mathrm{B}$ in the image. The algorithm for white balance mainly estimates the color temperature of the light source based on the input image, calculates the gain value of each color channel, and finally adjusts the gain value to correct the image. In this approach, the image can present the color effect under the standard light source. The gray world algorithm (GWA) was widely used; it equally divides the statistical values of $R, G$, and $B$ of each color channel component of the image collected by the camera.

The implementation steps of the algorithm were shown as follows:

Step 1) Calculate the color mean

$$
\bar{R}=\frac{1}{N} \sum_{i=1}^{N} R_{i} \quad \bar{G}=\frac{1}{N} \sum_{i=1}^{N} G_{i} \quad \bar{B}=\frac{1}{N} \sum_{i=1}^{N} B_{i}
$$

where $N$ is the sum of input image pixels; $R_{i}, G_{i}, B_{i}$, and $B_{i}$ represent the color components of the i-th pixel of the image.

Step 2) Select the standard of gain coefficient correction

The mean value of the green component was used as the calibration value to calculate the gain of each channel:

$$
k_{r}=\frac{\overline{\text { Grey }}}{\bar{R}} \quad k_{g}=\frac{\overline{\text { Grey }}=\bar{G}}{\bar{G}} \quad k_{b}=\frac{\overline{\text { Grey }}}{\bar{B}}
$$

where $k_{r}, k_{\mathrm{g}}$, and $k_{b}$ are the gain value of each color channel.

Step 3) Recalculate the components of each color in the image

$$
\left\{\begin{array}{l}
C\left(R^{\prime}\right)=C(R) * k_{r} \\
C\left(G^{\prime}\right)=C(G) * k_{g} \\
C\left(B^{\prime}\right)=C(B) * k_{b}
\end{array}\right.
$$

where $R^{\prime}, G^{\prime}$, and $B^{\prime}$ are the color components after image correction; $R, G$, and $B$ are the color components prior to image correction.

The obtained picture was processed with three primary colors based on the dynamic white balance of the camera. The LumiCam 1300 luminance colorimeter and the camera used in this system were used to obtain the high and low bright color coordinates of each character of the test piece marked by the original manufacturer. The test was carried out three times with 30-minute intervals, and each test was repeated for more than 30 times, that is, 138 times. Combining the two modes of low and high luminance, the max error of camera detection accuracy was $6.08 \%$ and $0.4 \%$, which could not satisfy the requirement of secondary measurement accuracy of $5 \%$. 
Therefore, the algorithm for high and low luminance weighted traceability was used for the correction. The steps of color coordinate correction model were shown as follows: Step 1) Calculate the error coefficient in the table and correct the high and low bright $\mathrm{X}$ and $\mathrm{Y}$ coefficients, respectively.

Step 2) Add the color coordinates $x$ and $y$ after high and low luminance correction.

Step 3) Divide the added color coordinates by two to obtain the corrected color coordinates.

3.3 Luminance correction model of electronic gear lever In this exploration, CMOS industrial cameras were used to capture pictures to establish the RGB color model. The gray value of the image was calculated by the $R, G$, and $B$ values of the captured image:

$$
D(x, y)=0.299 R+0.587 G+0.114 B
$$

Under normal exposure conditions, the gray value $D(\mathrm{x}$, $y)$ of any position $(x, y)$ pixel on the image taken by CMOS camera was linear with the logarithms of camera exposure $H$, as shown in Eq. (5).

$$
D(x, y)=a \lg H+b
$$

where $a$ is the contrast coefficient, and $b$ is the undetermined coefficient.

The camera exposure $H$ was equal to the product of the image sensor surface illuminance $E$ and the exposure time $T$. The illuminance $E$ was controlled by the camera aperture. The exposure time $T$ could be determined by the camera shutter. The relationship was shown as follows:

$$
H=E \cdot T
$$

Obtained by simultaneous Eq. (4), (5) and (6):

$$
L(x, y)=\frac{4}{\pi \tau} \cdot \frac{F^{2}}{T} \cdot 10^{\left(\frac{D(x, y)-b}{a}\right)}
$$

Eq.(7) is the luminance measurement formula of the imaging type luminance meter, which represents the functional relationship between the image gray value $D(x, y)$ and the luminance value $L(x, y)$.

The analysis indicates that CMOS industrial cameras realized gray measurement. The luminance of the characters on electronic gear lever was measured in the laboratory, the environment was $(25 \pm 2){ }^{\circ} \mathrm{C}$, and the voltage of the power supply voltage source was $(13 \pm 0.2) \mathrm{V}$. The reference source consumed 30 minutes. However, the actual production site test time should be controlled within 16 seconds. The common processing time of camera capture and main control computer should be controlled within 11 seconds, considering loading and unloading. During the actual test, the LED lamp bead flashed, and the exposure time of the CMOS camera should be controlled within 10 milliseconds.

To benchmark the laboratory test standards, further establishing the luminance model of the electronic gear lever was necessary to realize the nonlinear mapping between the instantaneous exposure gray value and the luminance value after the temperature drift. The steps for establishing the luminance model of electronic gear lever are as follows:

Step 1) Obtain the high and low luminance gray values of the character image captured by the camera in the darkroom.

Step 2) Obtain the front and back luminance values of the electronic gear lever after temperature drift in the laboratory environment.

Step 3) Calculate the errors before and after the temperature drift of the electronic gear lever.

Step 4) Map the high and low luminance gray values to the luminance values.

Step 5) Calculate the error.

The data were analyzed with MATLAB. The data conformed to the exponential function relationship:

$$
L=a x^{\wedge} b
$$

where $L$ is the luminance value, $X$ is the gray value, and $a$ and $b$ are the parameters.

Fig. 3 shows the luminance model fitting curve. The high and low brightness model parameters of all characters were different. Table 1 shows the specific matching parameters.

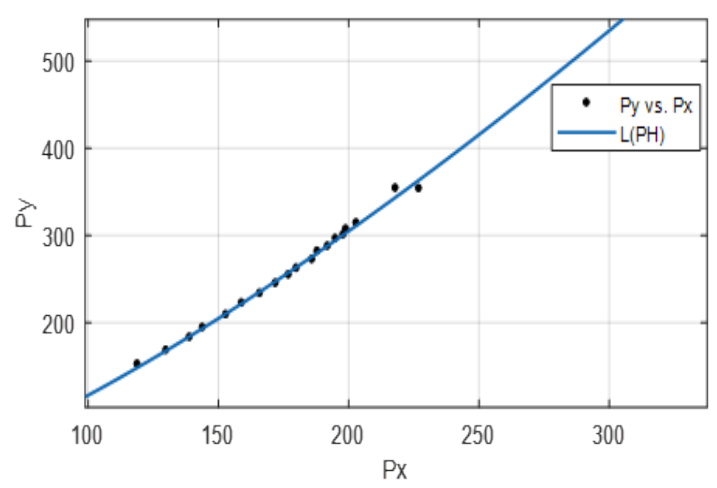

Fig. 3. Fitting curve of character $P$ high luminance

The relative error of the fitted data was relatively uniform. The average absolute relative deviation (AARD) of each character was less than $1 \%$. The maximum error of low luminance occurred in character $R$, and the maximum relative error of low(L) luminance was $3.1 \%$. The maximum highlight $(\mathrm{H})$ error occurred in the character, and the maximum relative error was $2.4 \%$. The results satisfied the $5 \%$ accuracy requirements of the secondary calibration equipment.

\subsection{Process of Fast Character Detection}

The shifter material affected the brightness. The production site had assembly abnormalities and manipulator transmission errors, resulting in various impurities on the shifter character surface and a certain rotation angle and inclination. This condition was difficult for character recognition. Through the establishment of an abnormal picture database (Fig. 4), the abnormal pictures were processed, and four types of defects were summarized by the cluster analysis. They were character defects, abnormal luminance values, abnormal color coordinates, and other abnormalities. The contents of character deformity included character contour deformity, edge black spots, internal black spots, bright spots, and serious inclination. 
Table 1. High and low luminance fitting coefficients and error statistics

\begin{tabular}{|c|c|c|c|c|c|c|c|c|c|}
\hline State & Data & $\mathrm{P}$ & $\mathrm{R}$ & $\mathrm{N}$ & $\mathrm{D}$ & $\mathrm{S}$ & + & - & $\nabla$ \\
\hline \multirow{5}{*}{$\mathrm{L}$} & $\mathrm{a}$ & 0.0507 & 0.0449 & 0.0420 & 0.0401 & 0.0500 & 0.0497 & 0.0495 & 0.0457 \\
\hline & b & 1.311 & 1.336 & 1.348 & 1.365 & 1.315 & 1.313 & 1.318 & 1.336 \\
\hline & $\min$ & $0.00 \%$ & $0.03 \%$ & $0.00 \%$ & $0.00 \%$ & $0.00 \%$ & $0.00 \%$ & $0.19 \%$ & $0.04 \%$ \\
\hline & $\max$ & $1.32 \%$ & $3.11 \%$ & $1.40 \%$ & $2.20 \%$ & $1.13 \%$ & $1.50 \%$ & $1.64 \%$ & $2.02 \%$ \\
\hline & AARD & $0.67 \%$ & $0.89 \%$ & $0.56 \%$ & $0.54 \%$ & $0.44 \%$ & $0.63 \%$ & $0.80 \%$ & $0.69 \%$ \\
\hline \multirow{5}{*}{$\mathrm{H}$} & $\mathrm{a}$ & 0.1988 & 0.2055 & 0.3075 & 0.1489 & 0.1902 & 0.228 & 0.2543 & - \\
\hline & $\mathrm{b}$ & 1.385 & 1.379 & 1.296 & 1.45 & 1.393 & 1.354 & 1.338 & - \\
\hline & $\min$ & $0.02 \%$ & $0.22 \%$ & $0.02 \%$ & $0.02 \%$ & $0.06 \%$ & $0.17 \%$ & $0.08 \%$ & - \\
\hline & $\max$ & $1.51 \%$ & $1.11 \%$ & $2.23 \%$ & $1.73 \%$ & $1.19 \%$ & $1.62 \%$ & $2.42 \%$ & - \\
\hline & AARD & $0.65 \%$ & $0.61 \%$ & $0.76 \%$ & $0.77 \%$ & $0.52 \%$ & $0.72 \%$ & $0.76 \%$ & - \\
\hline
\end{tabular}

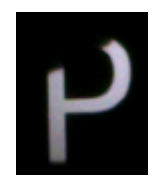

(a)

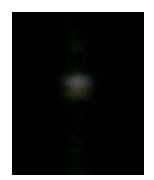

(d)

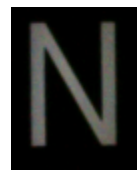

(g)

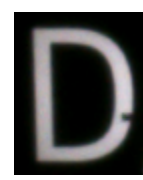

(b)

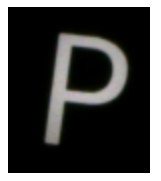

(e)

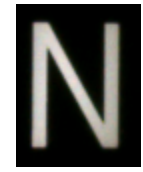

(h)

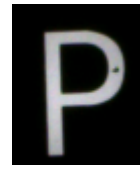

(c)

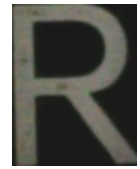

(f)

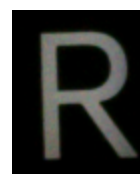

(i)
Fig. 4. Typical abnormal figures of incomplete characters (a) Incomplete. (b) Edge blackspot. (c)Internal black spot. (d) Highlights. (e)Severe tilt. (f)Uniformity anomaly. (g) Insufficient luminance. (h)Luminance abnormal. (i) Color coordinate abnormality

AI algorithms could not be used due to problems, such as fast iteration, evident incompleteness, and insufficient pictures of electronic gear lever. The traditional recognition algorithm was adopted. The character recognition process (Fig. 5) was divided into color coordinate correction, luminance correction, character positioning, character segmentation, feature extraction, and classification recognition. Character location was transformed into gray level, and Otsu algorithm was binarized to reduce the amount of algorithm operation; character segmentation was analyzed by eight adjacent connected domains; circle projection feature algorithm was used to extract feature information. After character recognition, curvature defect detection algorithm was used to judge the character deformity.

\section{(1) Improved method of circle projection feature}

The circumscribed circle template was connected with the minimum radius $R$ and the maximum radius $r$ of the characters of electronic gear lever. The $\mathrm{x}$-axis was the positive direction, the circle center $O$ was the origin to establish a polar coordinate system, and the eigenvalue $P(r)$ of the circle projection on the radius $r$ was obtained:

$$
\mathrm{P}(r)=\frac{1}{s} \sum_{\theta=0}^{2 \pi} T(r, \theta), 0 \leq r \leq R
$$

where $s$ is the number of pixels of a ring with a radius of $r$.

Furthermore, the circular projection vector group with continuously varying radius was obtained:

$$
\mathrm{P}=(P(0), P(1), \ldots, P(R))
$$

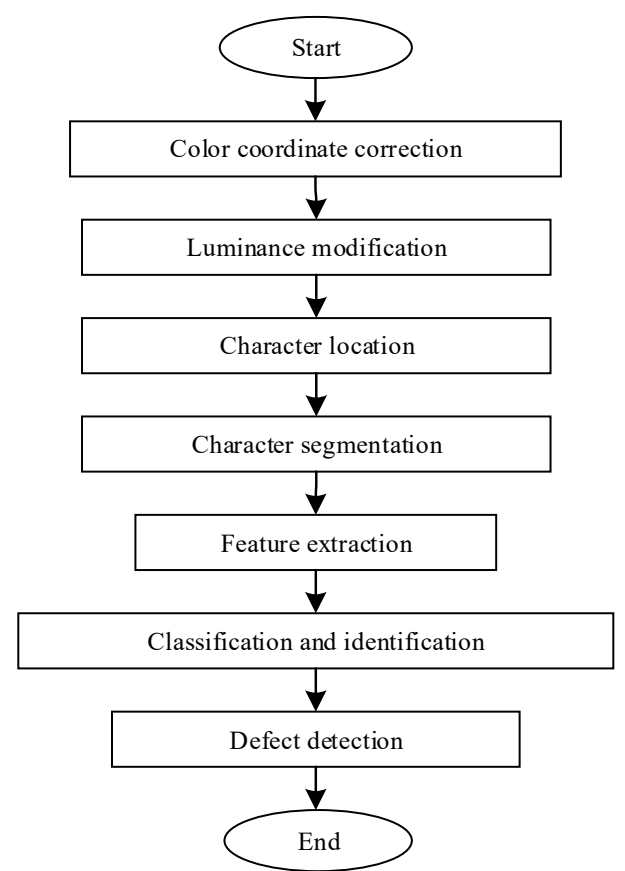

Fig. 5. Flow Diagram of Experiment

The standard algorithm for circle projection feature used a lossless circle projection with a continuous radius, which had complex calculations and high hardware requirements. $n$ equidistant circles were used for processing to improve the recognition speed. The minimum circumscribed circle radius of the character was $R$. The distance among the rings was $\frac{R}{n}$. The gray value of the character area was zero to 255 , and the average number of pixels on the ring with radius $R$ was considered the pixel mean characteristic value $G(r)$ of the character on radius $r$, which was composed of $n$ pixel mean characteristic values. The character pixel meant characteristic $G$, as follows:

$$
\begin{aligned}
& \mathrm{G}=\left(G\left(\frac{R}{n}\right), \ldots, G(r), \ldots, G(R)\right), G(r)=\frac{N(r)}{S(r)} \\
& \mathrm{N}(\mathrm{r})=\sum_{\theta=0}^{2 \pi} f(r, \theta), f(r, \theta)=\left\{\begin{array}{l}
0, T(r, \theta)=0 \\
1, T(r, \theta)=255
\end{array}\right.
\end{aligned}
$$

where $N(r)$ is the number of pixels with a gray value of 255 on a circle with a radius of $r . \mathrm{S}(\mathrm{r})$ is the number of pixels 
on a circle with a radius of $r$, $\frac{R}{\mathrm{n}} \leq\left(r=k^{*} \frac{R}{\mathrm{n}}\right) \leq R, k \in[1,2, \ldots, n]$.

The maximum and minimum values were calculated in the projection ring, and then the number characteristics of pixels were obtained by standardized processing. Finally, quantitative statistics was carried out.

\section{(2) Curvature detection}

The image was a 2D discrete function. The horizontal and vertical directions of the image were $\mathrm{x}$-axis and $\mathrm{y}$-axis, respectively, and the upper left vertex of the image was the starting origin.

The horizontal and vertical directions of the image were the $\mathrm{x}$-axis direction and the $\mathrm{y}$-axis direction, respectively, and the top left vertex of the image was the starting origin. The character edge curve point set $P(i)$ was obtained counterclockwise from the highest point of the character edge. $i$ was the point acquisition sequence value, $\mathrm{x}(i)$ was the abscissa of the edge point, and $\mathrm{y}(i)$ was the ordinate of the edge point. The set of character edge curve points is expressed as follows:

$$
\mathrm{P}(i)=\left[\begin{array}{lll}
x(0) & \ldots x(i) \ldots & x(m) \\
y(0) & \ldots y(i) \ldots & y(m)
\end{array}\right]
$$

The curvature calculation formula is as follows:

$$
\begin{aligned}
& X(i)=\lim _{\varepsilon \rightarrow 0} \frac{x(i+\varepsilon)-x(i-\varepsilon)}{2 \varepsilon} \\
& Y(i)=\lim _{\varepsilon \rightarrow 0} \frac{y(i+\varepsilon)-y(i-\varepsilon)}{2 \varepsilon} \\
& X(i)=\lim _{\varepsilon \rightarrow 0} \frac{x(i+\varepsilon)+x(i-\varepsilon)-2 x(i)}{\varepsilon^{2}}
\end{aligned}
$$

$Y(i)=\lim _{\varepsilon \rightarrow 0} \frac{y(i+\varepsilon)+\mathrm{y}(i-\varepsilon)-2 y(i)}{\varepsilon^{2}}$

$K=\frac{X(i) Y(i)-Y(i) X(i)}{(X(i)+Y(i))^{1.5}}$

The character edge curve in the image was a uniformly distributed discrete pixel point; thus, it was considered an integer value, and the step size $\Delta \mathrm{K}$ was further used instead of $\varepsilon . \Delta \mathrm{K}$ was an integer, and the curvature was calculated by the difference in the pixel coordinates on the curve, as follows:

$$
\begin{aligned}
& X(i)=\frac{x(i+\Delta K)-x(i-\Delta K)}{2 \Delta \mathrm{K}} \\
& Y(i)=\frac{y(i+\Delta K)-y(i-\Delta K)}{2 \Delta \mathrm{K}} \\
& X(i)=\frac{x(i+\Delta \mathrm{K})+x(i-\Delta K)-2 x(i)}{\Delta K^{2}} \\
& Y(i)=\frac{y(i+\Delta \mathrm{K})+\mathrm{y}(i-\Delta K)-2 y(i)}{\Delta K^{2}}
\end{aligned}
$$

\section{Result Analysis and Discussion}

\subsection{Statistical analysis of luminance value}

The luminance detection data of 4000 items were counted, and 40 electronic gear levers were sampled for comparison. Table. 2 shows the statistical results. The AARD satisfied the requirements of secondary measuring instruments, but the product was prone to abnormalities in the highlighted state.

Table. 2. Comparison table of luminance value of product sampling inspection

\begin{tabular}{c|c|c|c|c|c|c|c|c|c|c}
\hline State & Data & P & R & N & D & S & + & - & $\nabla$ \\
\hline & $\max$ & $1.31 \%$ & $1.03 \%$ & $3.54 \%$ & $2.28 \%$ & $1.03 \%$ & $2.47 \%$ & $2.24 \%$ & $3.96 \%$ \\
$\mathbf{L}$ & $\min$ & $0.00 \%$ & $0.00 \%$ & $1.30 \%$ & $0.18 \%$ & $0.07 \%$ & $0.47 \%$ & $0.10 \%$ & $0.00 \%$ \\
& AARD & $0.55 \%$ & $0.39 \%$ & $2.02 \%$ & $1.30 \%$ & $0.42 \%$ & $1.57 \%$ & $0.65 \%$ & $2.66 \%$ \\
\hline & $\max$ & $3.01 \%$ & $4.11 \%$ & $9.33 \%$ & $5.81 \%$ & $2.34 \%$ & $6.66 \%$ & $5.80 \%$ & - \\
$\mathbf{H}$ & $\min$ & $0.04 \%$ & $0.03 \%$ & $0.14 \%$ & $2.02 \%$ & $0.08 \%$ & $0.66 \%$ & $0.17 \%$ & - \\
& AARD & $0.91 \%$ & $0.88 \%$ & $1.84 \%$ & $3.23 \%$ & $0.76 \%$ & $2.12 \%$ & $1.62 \%$ & - \\
\hline
\end{tabular}

Table 3. Statistics of scale changes of different characters (pixels)

\begin{tabular}{c|c|c|c|c|c|c|c|c|c|c|}
\hline Data & P & R & N & D & $/$ & S & + & - & \multicolumn{1}{|c}{} \\
\hline Max & $61 * 103$ & $71 * 99$ & $73 * 95$ & $69 * 89$ & $48 * 105$ & $57 * 93$ & $61 * 61$ & $50 * 12$ & $72 * 47$ \\
Min & $39 * 58$ & $41 * 58$ & $45 * 59$ & $41 * 54$ & $29 * 64$ & $35 * 57$ & $35 * 36$ & $30 * 10$ & $41 * 29$ \\
\hline
\end{tabular}

\subsection{Display of incomplete character outline}

One hundred images of electronic gear lever (900 characters) collected at the production site were analyzed by manual experiments. Table 3 shows the statistical results. The characters in the test data set had scale and rotation changes, and the scale size change rate was close to $100 \%$. Taking the "P" character pixel point statistics as an example, the height and width change ranges were 58-103 and 39-61, respectively.

The recognition accuracy of the algorithm was compared with the algorithm for circle projection matching, the method of SIFT feature, and the algorithm for $\mathrm{Hu}$ moment (Fig. 6). The improved algorithm for circle projection matching had evident and stable differences in similarity values. The algorithm stability was improved, and it was relatively easy to cause errors. The recognition accuracy of the recognized circle projection matching increased by $12 \%$, which was higher than the recognition accuracy of the classic SIFT and Hu moment algorithms.

Character deformity includes incomplete character outline, edge black spots, and serious inclination. The improved algorithm for circle projection feature realized the discrimination of inclined characters, and the inclination rate was calculated in combination with the pixel position. If the inclination angles of all characters were the same, then the electronic gear lever was normal; otherwise, the characters 
were abnormal. Incomplete contours and internal black dots were distinguished by the number of connected domains. The difficulty of character defect detection lied in edge defect detection. The algorithm for curvature detection defected inner and outer contour black spots.

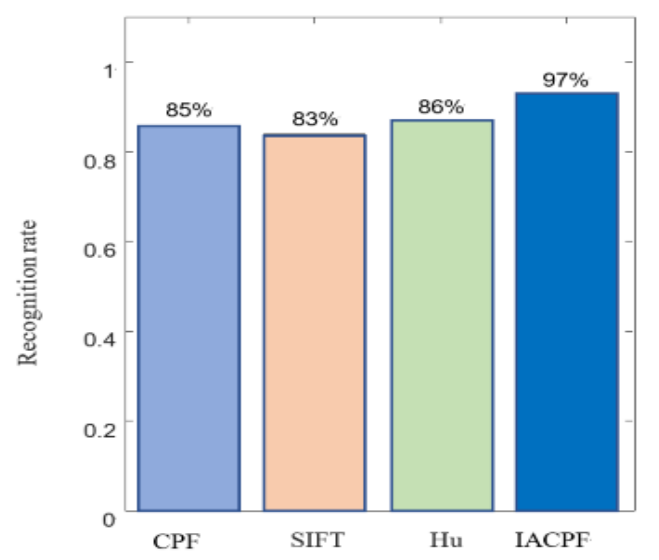

Fig. 6. Comparison of recognition algorithms

Taking the character $P$ as an example, the contour defect analysis was carried out. Fig. 7 shows the curvature diagram and curvature marking diagram of the outer edge of the incomplete $P$ character with steps two, six, and 10 and thresholds of $0,0.15$, and 0.25 , respectively. When $\Delta \mathrm{K}$ was two, detecting a fixed number of continuous corners using the threshold value was difficult due to the influence of local breaking points. It was not conducive to defect detection. When $\Delta \mathrm{K}$ was six and the threshold value was 0.15 , normal continuous corners could be detected, and a defect in the character was evident. If the threshold was 0.25 , then some corners and the curvature marked near the defect position disappeared, and the character incompleteness was detected. When $\Delta \mathrm{K}$ was 10 , the straight-line part was better than zero because the step length was extremely large. Selecting an appropriate threshold for incomplete detection was difficult. Therefore, when $\Delta \mathrm{K}$ was extremely small, then it was affected by noise points and breaking points, and the character was judged according to the degree of curvature. When $\Delta \mathrm{K}$ was extremely large, then the curvature value extends longer, which was not conducive to the selection of the threshold. An excessively large threshold also affected the normal judgment of incompleteness with a suitable step size.

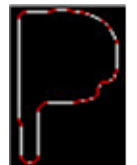

(a)

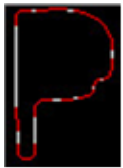

(d)

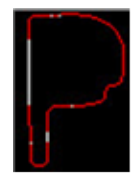

(g)

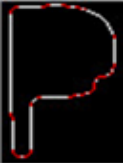

(b)

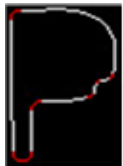

(e)

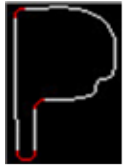

(h)

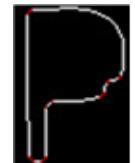

(c)

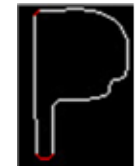

(f)

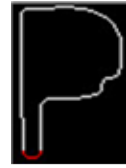

(i)
Fig. 7. Comparison of character $P$ incomplete curvature detection. (a) $\Delta k=2, T=0$. (b) $\Delta k=2, T=0.15$. (c) $\Delta k=2, T=0.25$. (d) $\Delta k=6, T=0$. (e) $\Delta \mathrm{k}=6, \mathrm{~T}=0.15$. (f) $\Delta \mathrm{k}=6, \mathrm{~T}=0.25$. (g) $\Delta \mathrm{k}=10, \mathrm{~T}=0$. (h) $\Delta \mathrm{k}=10, \mathrm{~T}=0.15$. (i) $\Delta \mathrm{k}=10, \mathrm{~T}=0.25$

Fig. 8 shows the edge curvature mark diagram of $\mathrm{R}, \mathrm{N}$, $\mathrm{D}, \mathrm{S}, /, \nabla,+$ and - missing characters when the curvature step length of $\mathrm{P}, \mathrm{R}, \mathrm{N}$, and $\mathrm{D}$ characters was six, the curvature step length of $\mathrm{S}$ and / was four, the curvature step length of $\nabla,+$ and - was three, and the curvature threshold was 0.15 . The intuitive analysis of the curvature algorithm detected the edge defects of each character.

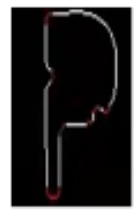

(a)

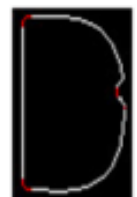

(d)

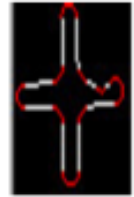

(g)

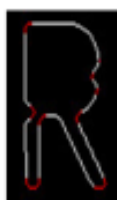

(b)

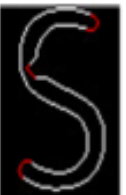

(e)

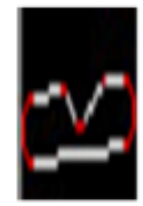

(h)

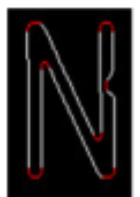

(c)

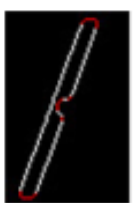

(f)

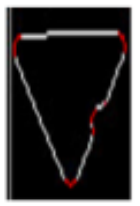

(i)
Fig. 8. Curvature detection results of outer contour defect. (a) P. (b) R (c) N. (d) D. (e) S. (f) /. (g) +. (h) -. (i) $\nabla$

\section{Conclusions}

This study started with the temperature drift traceability, established the color coordinate and luminance model for transient detection of the electronic gear lever, and analyzed the incomplete character contour recognition to replace the manual visual inspection method to improve the contour recognition rate and detection efficiency. Finally, the following conclusions could be drawn:

(1) The character luminance tended to be stable after 30 minutes of temperature drifting test in the laboratory. The change in luminance value showed nonlinear attenuation, and the attenuation degree of each character was different.

(2) The gray value obtained by transient measurement of a character had an exponential function relationship with the luminance value after temperature drifting, and the high and low luminance coefficients of the same character were different.

(3) The improved circular projection feature method had good robustness to surface character recognition with a certain rotation angle. It recognized the character contour with a change rate of 100 .

(4) The curvature detection algorithm detected the incomplete contour of characters, and the step size varied with the characters. However, the threshold was uniform.

On the basis of the picture analysis of the transient state of the light off characteristics of the electronic gear lever in the production environment, the transient color coordinate and luminance model of the characters of the electronic gear lever was established. The proposed fast detection algorithm was reliable and stable. A reference for the function 
detection and development of other electronic gear levers or interior luminous display components is provided. We will modify the proposed model in the future to improve the recognition rate of contour incompleteness to avoid the influence of the limited number of images.
This is an Open Access article distributed under the terms of the Creative Commons Attribution License.

\section{References}

1. Wang T., "Development status and trend of China's automobile industry". Enterprise Reform and Management, (16), 2020, pp. 214-215.

2. SaoBo L., Jing Y., Zheng W., Shude Z., Guanchi Y., "Review on the development and application of defect detection technology". Acta Automatic Sinica, 46(11),2020, pp. 2319-2336

3. Bingkai L, Yudong L, Lin W., "Study of Dark Current Random Telegraph Signal in Proton-irradiated Backside Illuminated CMOS Image Sensors". Results in Physics, 19(44),2020, pp. 2020,

4. Ayumi O., Takeshi K., Ryosuke O., "Reduction of Dark Current in CMOS Image Sensor Pixels Using Hydrocarbon-molecular-ionimplanted Double Epitaxial Si Wafers". Sensors (Basel, Switzerland),20(22),2020, pp.512-530.

5. Wei D., Fossum Eric R., "1/ f Noise Modelling and Characterization for CMOS Quanta Image Sensors”. Sensors (Basel, Switzerland), 19(24),2019, pp. 1-16.

6. Bernacki J., "Automatic Exposure Algorithms for Digital Photography”. Multimedia Tools and Applications, 13(7),2020, pp. 321-327.

7. Zhihui G., Yuanbao G., Hongtao Y., “ Auto-exposure Algorithm Based on Luminance Histogram and Region Segmentation". Applied Mechanics and Materials, 30(82), 2014, pp. 2278-2282.

8. Sohaib A, Robles-Kelly A., "Exposure Time Calculation for Spectral Cameras". Journal of Electronic Imaging, 24(5),2015, pp. 10211031.

9. Bai Y., "Research on Auto-exposure Algorithm Based on Image Big Data and Information Entropy". Modern Electronic Technology, 3(2), 2019, pp. 201-208.

10. Limin L., Hao P., Bin D., Mingzhi X., "Brightness detection and correction algorithm of CCD camera full-color LED display".
Journal of Huazhong University of Science and Technology (Natural Science Edition), 44(8),2016, pp. 75-79.

11. Huang C., Zhu J.S., LI Z. R., Zhu J.L., "Luminance measurement of ship night light Environment based on color CCD camera". Photoelectric Engineering, 45(7), 2018, pp. 10-15.

12. Tang J. L., Zhang C., Guo Y. F., Su B. H., Su Q. L., "Color correction algorithm for color image based on multi-color spatial information". Computer Science, 47(21),2020, pp. 157-160+165.

13. Huang C. Q., "Study on intelligent white balance based on deep convolutional neural network". Journal of Optoelectronics. laser, 31(12), 2020, pp. 278-1287.

14. Silva S M, Jung C., "Real-Time Brazilian License Plate Deception and Recognition Using Deep Convolutional Neural Networks". In: Proceedings of the 30th SIBGRRAPI-Conference on Graphics, Patterns and Images, Niteroi, Brazil: IEEE, 2017, pp.55-62.

15. Lee S., Song K., Park, J., "Car plate recognition based on CNN using embedded system with GPU”. In: 2017 International Conference on Human System Interactions, Ulsan, South Korea: IEEE, 2017, pp.239-241.

16. Yan L., Hongbo Z., Lianying S., "License plate recognition method based on SIFT feature matching". Computer Engineering and Applications, 52(12), 2016, pp. 194-200.

17. Shengping W., Zhefeng F., Peifeng H., Shihua Z., "Research on AGV character recognition based on improved $\mathrm{Hu}$ moment algorithm". Computer Measurement and Control, 28(05),2020, pp. 229-232+251.

18. Yihsien L., Chinhsing C., "Template matching using the parametric template vector with translation, rotation and scale invariance". Pattern Recognition, 41(7), 2008, pp: 2413-2421. 